Fig. S5A. Western blotting of commercial Indian antivenoms against the venoms of pan-Indian populations of $N$. naja.

SDS-PAGE

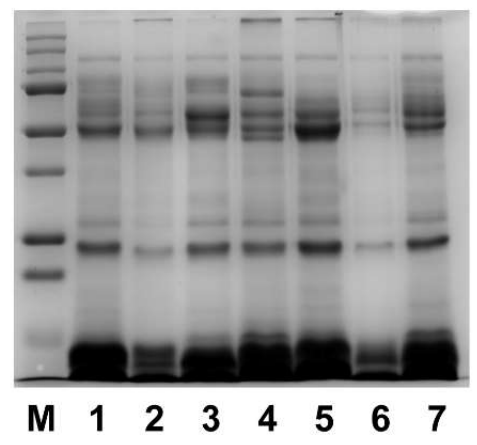

\section{Premium Serums}

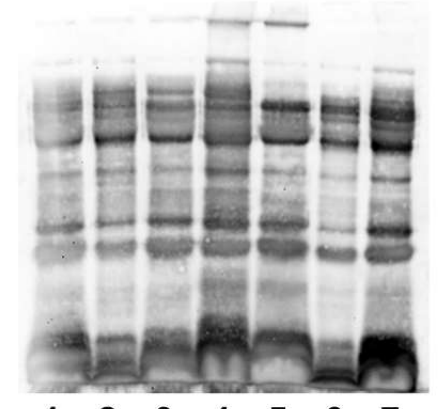

$\begin{array}{lllllll}1 & 2 & 3 & 4 & 5 & 6 & 7\end{array}$

Bharat Serums

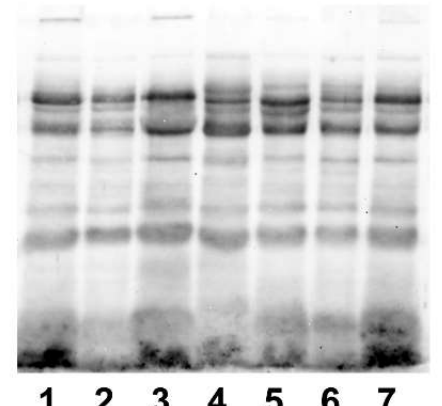

\section{Naive IgG}

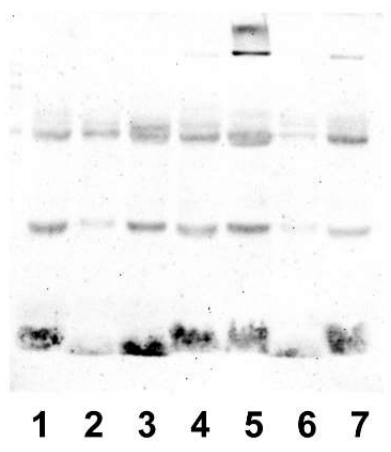

VINS

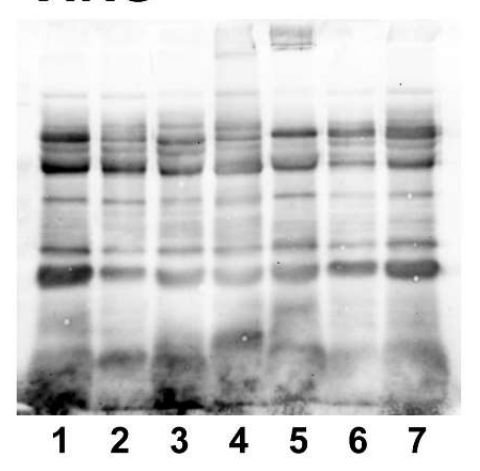

Haffkine

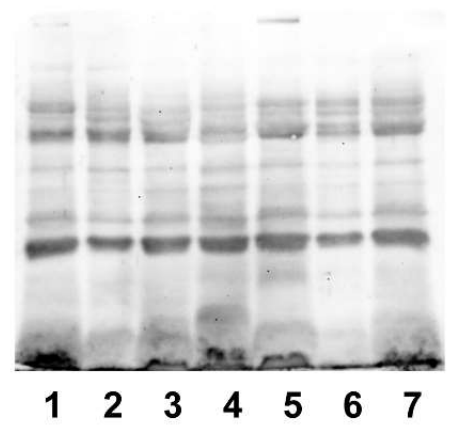

This figure depicts the binding affinities (western blots) of commercial Indian antivenoms against the venoms of biogeographically distinct populations of $N$. naja. M. Marker; 1. PB (north India); 2. TN (south India); 3. AP (southeast India); 4. WB (east India); 5. RJ (west India); 6. MH (southwest India); and 7. MP (central India). 
Fig. S5B. Heatmap of venom recognition potential of commercial Indian antivenoms against the venoms of pan-Indian populations of $N$. naja.

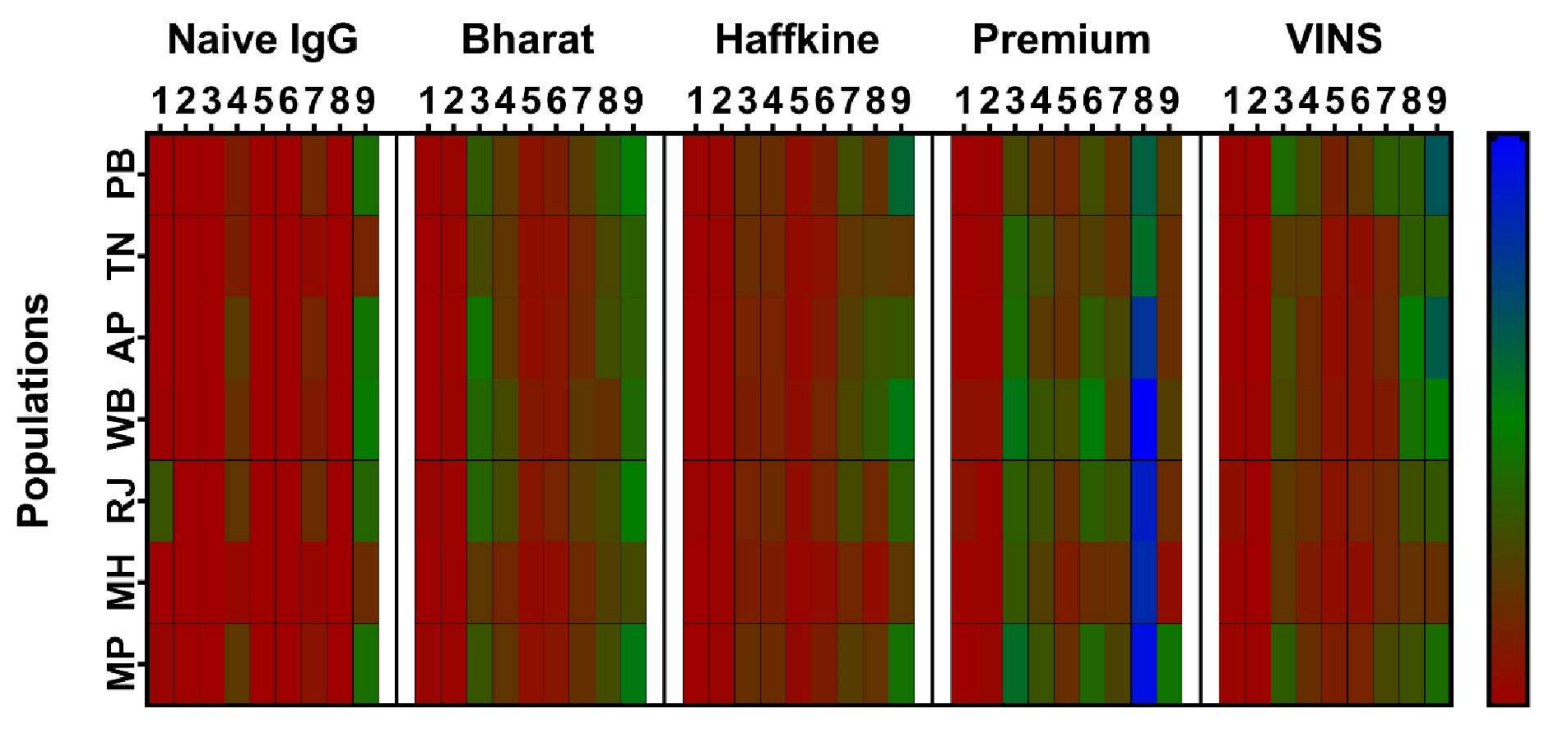

Binding efficiency at dilution 1:200

This figure depicts the binding affinities of commercial Indian antivenoms and naive horse IgGs against the $N$. naja venoms as a heatmap, on a gradient scale of red (low binding) to blue (high binding). The values were determined for individual immunoblot bands (1 to 9) using densitometric analysis. Populations: PB (north India); TN (south India); AP (southeast India); WB (east India); RJ (west India); MH (southwest India); and MP (central India). This heatmap was generated based on the densitometric analysis of immunoblot bands using ImageJ. 